\title{
Differential diagnosis of leukoplakia versus lichen planus of the oral mucosa based on digital texture analysis in intraoral photography
}

\author{
Kamil Jurczyszyn ${ }^{1, A, B, D}$, Marcin Kozakiewicz ${ }^{2, A, C, D}$ \\ ${ }^{1}$ Department of Oral Surgery, Wroclaw Medical University, Poland \\ ${ }^{2}$ Department of Maxillofacial Surgery, Faculty of Military Medicine, Medical University of Lodz, Poland \\ A - research concept and design; $B$ - collection and/or assembly of data; $C$ - data analysis and interpretation; \\ $D$ - writing the article; $E$ - critical revision of the article; $F$ - final approval of the article
}

\section{Address for correspondence}

Kamil Jurczyszyn

E-mail: kjurczysz@interia.pl

\section{Funding sources}

Medical University of Lodz: grant No. 503/5-061-

02/503-51-001 and 503/5-061-02/503-51-002,

Wroclaw Medical University: grant No. PBMN-152

\section{Conflict of interest}

None declared

Received on December 26, 2018

Reviewed on January 31, 2019

Accepted on February 18, 2019

Published online on March 20, 2019

Cite as

Jurczyszyn K, Kozakiewicz M. Differential diagnosis of leukoplakia versus lichen planus of the oral mucosa based on digital texture analysis in intraoral photography. Adv Clin Exp Med. 2019;28(11):1469-1476.

doi:10.17219/acem/104524

DOI

10.17219/acem/104524

\section{Copyright}

Copyright by Author(s)

This is an article distributed under the terms of the

Creative Commons Attribution Non-Commercial License

(http://creativecommons.org/licenses/by-nc-nd/4.0/)

\begin{abstract}
Background. A noninvasive, accurate and quick diagnosis is very important to general practitioners and specialists who care for the health of patients' oral cavity mucosa. The main enemies are precancerous lesions: leukoplakia and lichen planus (LP).

Objectives. The aim of this study was to attempt to formulate a differential diagnosis for leukoplakia vs LP in the oral mucosa based on digital texture analysis in intraoral macrophotography.

Material and methods. The study was comprised of 21 patients affected by leukoplakia, 21 affected by LP and 21 healthy volunteers. Intraoral photography of all participants was taken perpendicularly to the buccal mucosa. To achieve the maximum possible contrast, a high-pass filter was applied and level tools were then used to equalize the histograms of the images. After that, the images were converted into 8-bit grayscale. Two features of run length matrix and 2 of co-occurrence matrix were used for texture analysis. Analysis of variance (ANOVA) was used to check for differences. Factor analysis (FA) and classification with artificial neural network (ANN) were performed.
\end{abstract}

Results. The results revealed a simple possible differentiation of both types of precancerous lesions from normal mucosa $(p<0.05)$. Factor analysis and ANN can help in differentiating the 3 study groups from one another.

Conclusions. Differential diagnosis of leukoplakia and LP in the oral mucosa based on digital texture analysis in intraoral macrophotography is possible. It can be used to develop smartphone applications and can be also a helpful tool for general dentists to define the clinical problem before a consultation with a specialist.

Key words: lichen planus, leukoplakia, texture analysis, oral mucosa pathology 


\section{Introduction}

Cancer of the oral mucosa is often preceded by premalignant lesions. Leukoplakia and lichen planus (LP) in the oral mucosa are known to pose an increased risk of malignant transformation ( $5 \%$ and $2 \%$, respectively). The etiology varies in these disorders, but both present as white lesions in the mucous membrane., ${ }^{1,2}$ The World Health Organization (WHO) definition of leukoplakia from 2005 characterizes it as "a white plaque of questionable risk having excluded (other) known diseases or disorders that carry no increased risk for cancer". ${ }^{3}$ The etiology of leukoplakia is multifactorial. The most important risk factors are cigarette smoking, alcohol consumption, poor oral hygiene, electrogalvanic currents (due to various metals in the oral cavity, i.e., gold, amalgam and nickel), and irritation caused by food. Lichen planus is a relatively common chronic inflammatory mucocutaneous disease. It is believed to result from an abnormal T-cell-mediated immune response in which basal epithelial cells are recognized as foreign because of changes in the antigenicity of their cell surface. ${ }^{4}$ Both antigen-specific and non-specific mechanisms are thought to be involved in the pathogenesis of oral LP. ${ }^{2}$

Due to the increased risk of malignant transformation, it is important to diagnose and distinguish between oral leukoplakia and LP early on. The main feature of leukoplakia and LP is hyperkeratosis of the epithelium. Both of these lesions have a very irregular shape. The heterogenic form of leukoplakia is clinically similar to the erosive form of LP, so biopsy and histopathological examination are still the golden standard during the diagnosis process.

The clinical perspective focuses on the importance of a noninvasive, accurate and timely diagnosis. Such attempts have been made in dermatology and oral surgery, leading to the conclusion that manual segmentation by general practitioners is feasible in the described computer-aided diagnostic system for classifying benign and malignant skin lesions. ${ }^{5,6}$ To date, no studies have been published on that topic for oral mucosa diagnosis and automated image segmentation.

Texture analysis is used during computed tomography (CT) analysis in the case of bone and soft tissue lesions, but there are no publications about the application of texture analysis in the differential diagnosis of leukoplakia vs LP in the oral mucosa. ${ }^{7,8}$

The aim of this study is to attempt to formulate a differential diagnosis of leukoplakia vs LP in the oral mucosa based on digital texture analysis in intraoral macrophotography.

\section{Material and methods}

\section{Patients}

Twenty-one patients affected by leukoplakia (11 females and 10 males) and 21 affected by LP (16 females and 5 males) were included in this study. All lesions were histopathologically verified (with standard hematoxylin and eosin (H\&E) staining) on specimens taken from pathological oral mucosa under local anesthesia. The control group consisted of 21 healthy volunteers. The mean age of the study group was 58 years.

Intraoral photography of normal oral mucosa, LP and leukoplakia were taken with a Canon EOS 500D digital camera (Canon, Ōta, Tokyo, Japan) with a $13 \mathrm{~mm}$ macro ring and a $50 \mathrm{~mm}$ lens at $\mathrm{f} 1.8$ (Canon), and a YN-14EX ring flashlight (Yongnuo Photographic Equipment, Shenzhen, China).

All procedures were conducted after obtaining the approval of the Ethics Committee of Wrocław Medical University, Poland (approval No. KB-367/2014).

\section{Image preprocessing}

All of the graphical operations were performed in GIMP v. 2.10.8 (GNU Image Manipulation Program; www.gimp. org). In the center of the lesion, a square $300 \times 300$ pixels in size was selected. These selected portions were cropped from the original photos. To achieve the maximum possible contrast, a high-pass filter was applied and level tools were then used to equalize the histograms of the images. After that, the images were converted into 8-bit grayscale. The files were saved in TIFF format without any compression algorithms. All graphical operations are presented in Fig. 1, while Fig. 2 shows the clinical photographic material of leukoplakia, LP and normal mucosa.

\section{Texture analysis}

If $p(i, j)$ is the number of times when there is a run of length $j$ with a gray level of $i, N g$ is the number of gray levels and $N r$ is the number of runs, ${ }^{9}$ then definitions of the parameters of the run-length matrix $p(i, j)$ are given below.

Long run emphasis inverse moments (LngREmph):

$$
\text { LngREmph }=\left(\sum_{i=1}^{N_{g}} \sum_{j=1}^{N_{r}} j^{2} p(i, j)\right) / C
$$

Short run emphasis inverse moments (ShrtREmph):

$$
\text { ShrtREmph }=\left(\sum_{i=1}^{N_{g}} \sum_{j=1}^{N_{r}} \frac{p(i, j)}{j^{2}}\right) / C
$$

The coefficient $C$ :

$$
C=\sum_{i=1}^{N_{g}} \sum_{j=1}^{N_{r}} p(i, j)
$$

The second-order histogram is known as the co-occurrence matrix $h d \theta(i, j) .{ }^{10}$ When divided by the total number of neighboring pixels $R(d, \theta)$ in region of interest (ROI), this matrix becomes an estimate of the joint probability

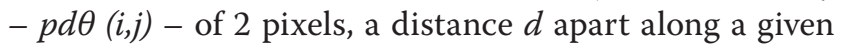



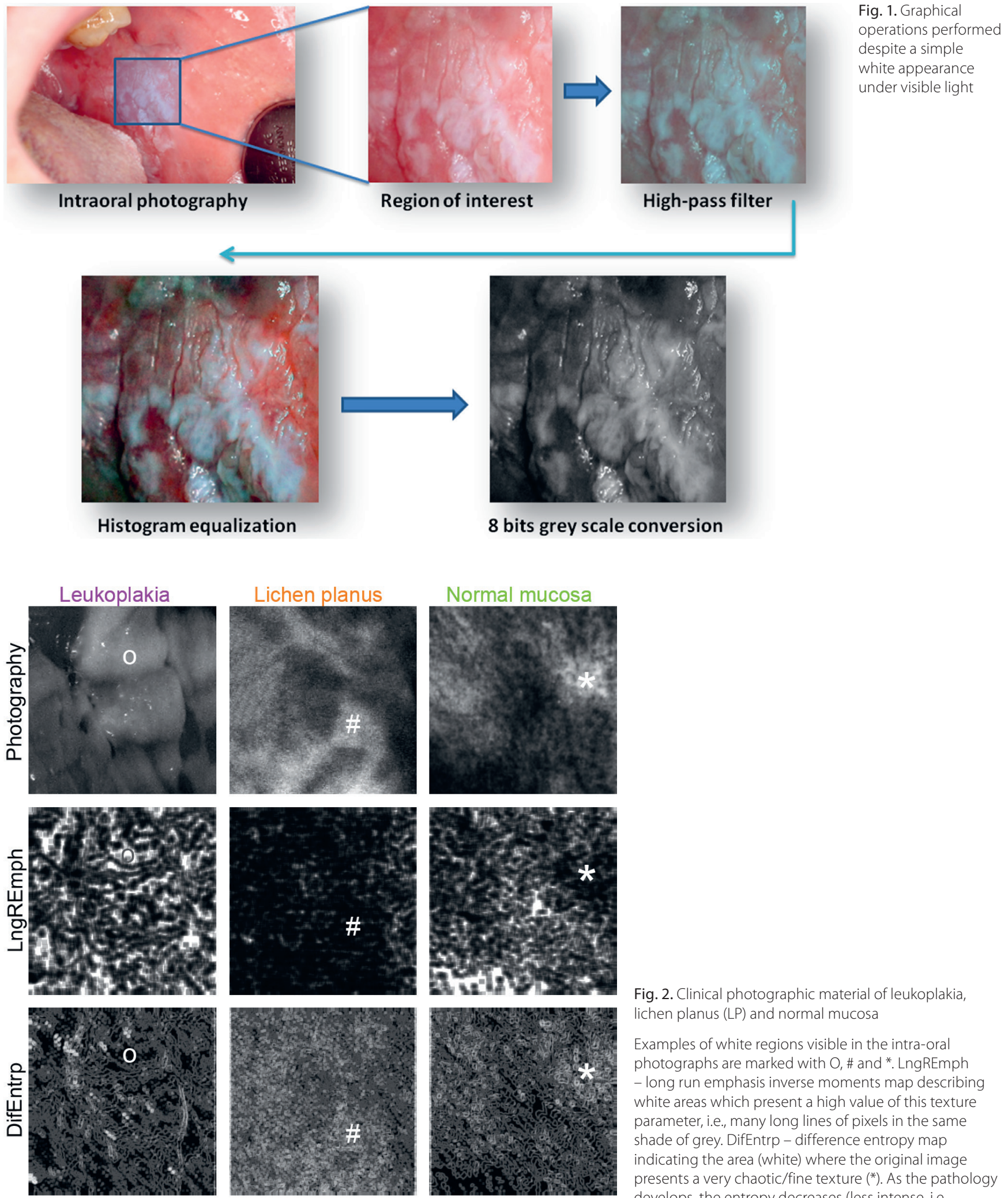

Fig. 2. Clinical photographic material of leukoplakia, ichen planus (LP) and normal mucosa

Examples of white regions visible in the intra-oral photographs are marked with $\mathrm{O}$, \# and *. LngREmph - long run emphasis inverse moments map describing white areas which present a high value of this texture parameter, i.e., many long lines of pixels in the same shade of grey. DifEntrp - difference entropy map indicating the area (white) where the original image presents a very chaotic/fine texture $\left(^{*}\right)$. As the pathology develops, the entropy decreases (less intense, i.e.,
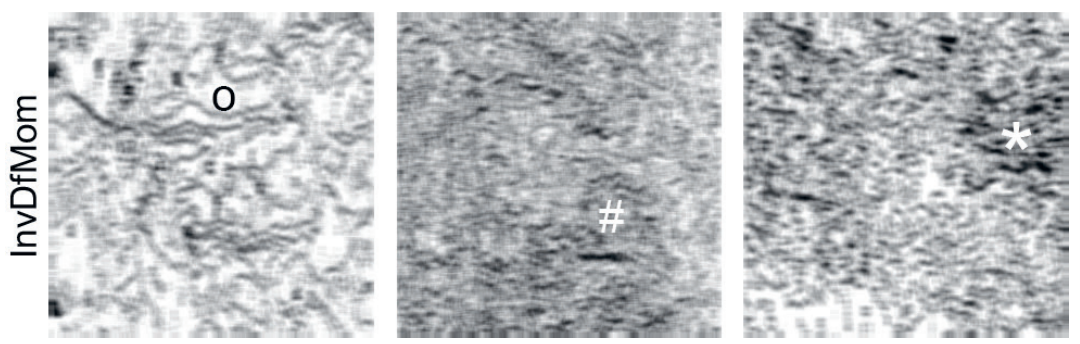
a darker appearance on the map): a minor decrease in LP (\#) and a major decrease in leukoplakia (O). InvDfMom - inverse difference moment map describing white areas which present a monotonic texture, contrary to black areas where the original image has a rich texture, i.e., a fine structure; that fine structure is lost in leukoplakia and LP. Note: texture analysis of the observed white regions in normal mucosa photography $\left(^{*}\right)$ reveals different InvDfMom results (less intense) than white regions in leukoplakia $(O)$ and LP. 
direction $\theta$, having the particular (co-occurring) values $i$ and $j$. Formally, given the function $f(x, y)$ with a set of $N g$ discrete intensity levels, the matrix $h d \theta(i, j)$ is defined such that its $(i, j)$ th entry is equal to the number of times that

$$
f\left(x_{1}, y_{1}\right)=i \text { and } f\left(x_{2}, y_{2}\right)=j
$$

where $\left(x_{2}, y_{2}\right)=\left(x_{1}, y_{1}\right)+(\mathrm{d} \cos \theta, \mathrm{d} \sin \theta)$.

This yields a square matrix whose dimension is equal to the number of intensity levels in the image, for each distance where $d=5$ pixels and orientation with angles $\theta=0^{\circ}, 45^{\circ}, 90^{\circ}$, and $135^{\circ}$ (such angles are considered and then their average is calculated to combine spatial information into single number). Reducing the number of intensity levels (through quantization) helps to remove noise, with some loss of textural information (as low as 4-bit in this case). The co-occurrence matrix-derived parameters are defined by the equations that follow, where $p x(i)$ and $p y(j)$ are the marginal distributions.

Difference entropy (DifEntrp):

$$
\text { DifEntrp }=-\sum_{i=1}^{N_{g}} p_{x-y}(i) \log \left(p_{x-y}(i)\right)
$$

Inverse difference moment (InvDfMom):

$$
\text { InvDfMom }=\sum_{i=1}^{N_{g}} \sum_{j=1}^{N_{g}} \frac{1}{1+(i-j)^{2}} p(i, j)
$$

The calculations were performed in Mazda v. 4.6 software (Lodz University of Technology, Poland) on selected features. ${ }^{11-15}$ The differences among the 3 study groups (normal mucosa, LP and leukoplakia) were checked using one-way analysis of variance (ANOVA). Next, in order to obtain the single factor which would account for most of the variability among the 2 variables (difference entropy and inverse difference moment) factor analysis (FA) was done. The principal component method was used. Thus, the initial communality estimates were set to assume that all of the variability in the data is due to this common factor. This factor was named the co-occurrence factor due to inner information extracted from 2 co-occurrence matrix features.

The efficacy of automated diagnosis was checked using a neural network Bayesian classifier (Fig. 3). This procedure used a probabilistic neural network (PNN) to classify cases into different diagnoses, based on 3 input variables: long run emphasis inverse moments, short run emphasis inverse moments and the co-occurrence factor. All of the statistical analyses were performed with Statgraphics Centurion XVI software (StatPoint, The Plains, USA).

\section{Results}

No differences between LP and leukoplakia were found in any of the investigated features. Both lesions presented very significant differences from the reference oral mucosa in difference entropy and inverse difference moment (Fig. 4). The results are presented in Table 1.

In the factor analysis one factor was extracted (given name: co-occurence factor), since only 1 factor had

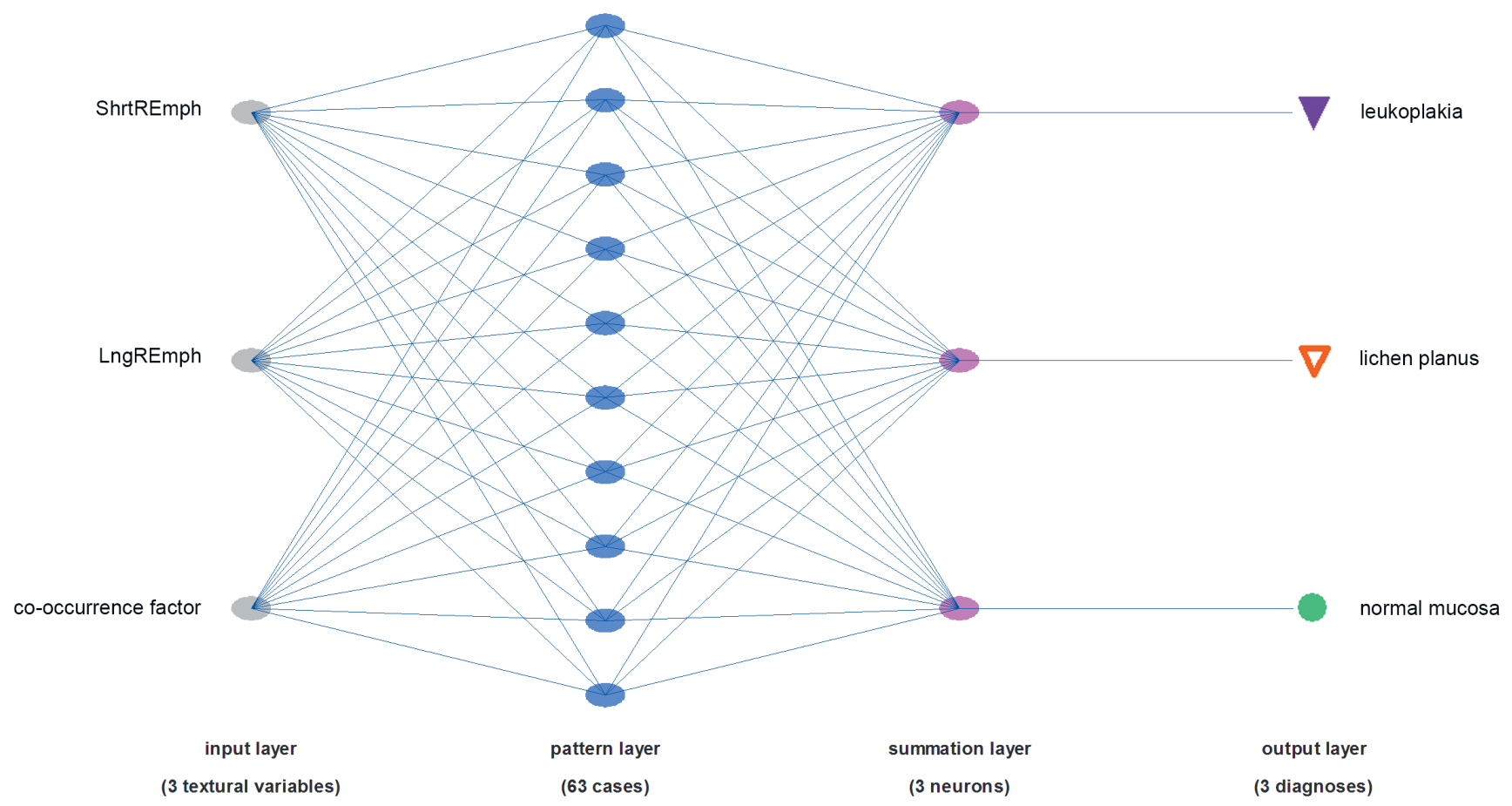

Fig. 3. Schemes of the artificial neural network (ANN) used for automated differential diagnosis of leukoplakia, lichen planus (LP) or normal mucosa in intra-oral photographic imagery. Input data include run length matrix features (ShrtREmph and LngREmph) and co-occurrence matrix features (1 factor extracted from 2 strong textural features: difference entropy (DifEntrp) and inverse difference moment (InvDfMom); the factor name is named the cooccurrence factor) 
Table 1. Summary statistics of textural features in normal oral mucosa, lichen planus and leukoplakia lesions. The 2 pathological lesions cannot be diagnosed differentially from one another

\begin{tabular}{|l|c|c|c|c|}
\multicolumn{1}{|c|}{ Textural feature } & $\begin{array}{l}\text { Normal } \\
\text { mucosa }\end{array}$ & $\begin{array}{c}\text { Lichen } \\
\text { planus }\end{array}$ & $\begin{array}{c}\text { Leukoplakia } \\
\text { lesion }\end{array}$ \\
\hline Long run emphasis inverse moment (LngREmph) & $10.84 \pm 13.86^{*}$ & $18.11 \pm 18.32^{*}$ & $13.84 \pm 9.42^{*}$ \\
vs normal oral mucosa
\end{tabular}

No differences were found between lichen planus and leukoplakia; ANOVA - analysis of variation; * lack of normal distribution; \# factor composed of difference entropy and inverse difference moment.

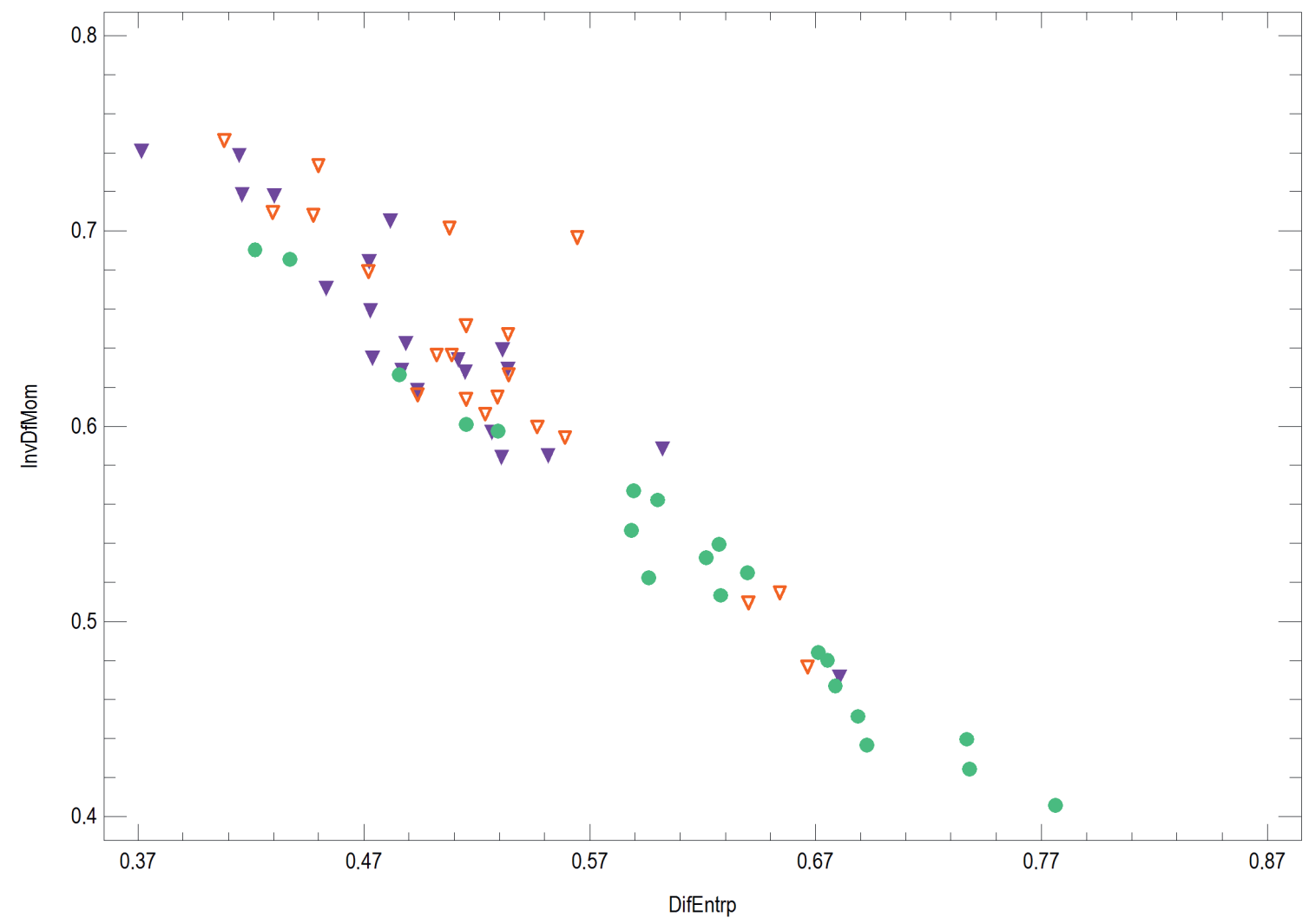

Fig. 4. The co-occurrence matrix parameters used in this study were difference entropy (DifEntrp) and inverse difference moment (InvDfMom). Both parameters clearly differentiate pathological lesions from normal mucosa ( $p<0.0001)$, but cannot help in distinguishing leukoplakia from lichen planus ( $\mathrm{LP}$ ) (Table 1)

an eigenvalue greater than 1.0. This factor accounted for $97.9 \%$ of the variability of difference entropy and inverse difference moment (InvDfMom) in the original data. Both textural features can be used very reliably $(\mathrm{p}<0.0001)$ for diagnosing leukoplakia and LP of the oral mucosa. The equation of the calculated factor is:

$$
\begin{gathered}
\text { Co }- \text { occurrence factor }= \\
=0.989253 \text { DifEntrop }-0.989253 \text { InvDfMom, }
\end{gathered}
$$

where the values of the variables of the equation are standardized by subtracting their means and dividing by their standard deviations (SDs). It also shows the estimated communalities, which can be interpreted as estimating the proportion of variability in each variable which is attributable to the extracted factor. Both types of lesions showed a highly significant difference from the reference mucosa as far as this factor was concerned (Table 1).

Out of the 63 cases in the training set, $61.9 \%$ were correctly classified using the neural network (Table 2 ). The reference oral mucosa was correctly classified in $90 \%$ of cases, LP in $38 \%$ and leukoplakia in $57 \%$. Based on the PNN, a correlation between short run emphasis inverse moment (ShrtREmph) and the co-occurrence factor was found (Fig. 5). Generally speaking, negative values of the co-occurrence factor combined with values of ShrtREmph higher than 0.3 describe a pathological lesion. When the ShrtREmph is $0.3-0.8$ and the co-occurrence factor is -0.2 , the lesion in the image should be classified as leukoplakia. 
Table 2. Results of probabilistic neural network (PNN) classifying cases into different diagnoses (normal oral mucosa, lichen planus and leukoplakia), based on 3 input variables: long run emphasis inverse moments, short run emphasis inverse moments and co-occurrence factor. Percent of training cases correctly classified: $61.9 \%$

\begin{tabular}{|l|c|c|c|c|}
\multicolumn{1}{|c|}{ Actual diagnosis } & Group size & Predicted leukoplakia & Predicted lichen planus & Predicted normal mucosa \\
\hline Leukoplakia & 21 & $12(57.14 \%)$ & $7(33.33 \%)$ & $2(9.52 \%)$ \\
\hline Lichen planus & 21 & $10(47.62 \%)$ & $8(38.10 \%)$ & $3(14.29 \%)$ \\
\hline Normal mucosa & 21 & $1(4.76 \%)$ & $1(4.76 \%)$ & $19(90.48 \%)$ \\
\hline
\end{tabular}

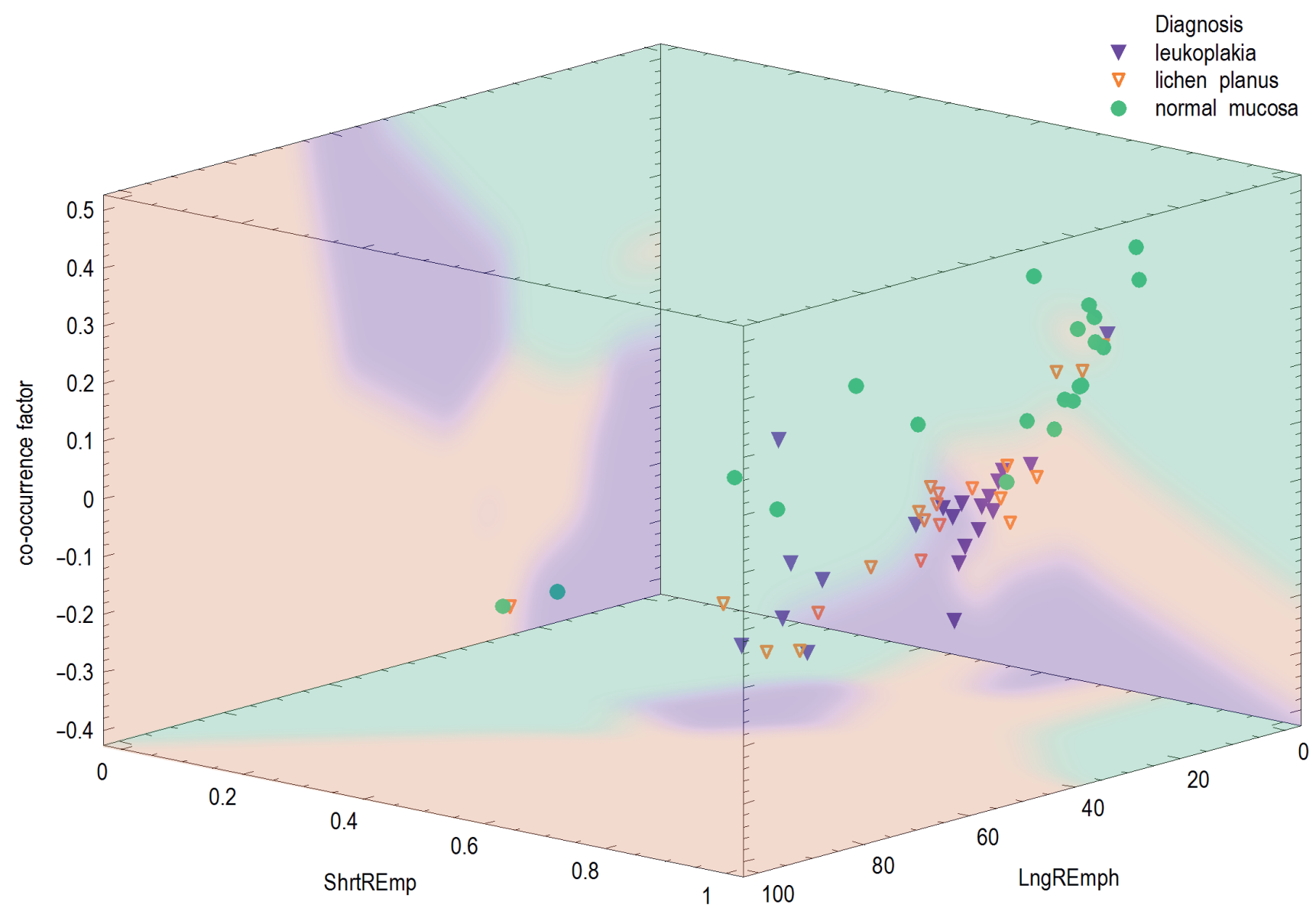

Fig. 5. The artificial neural network (ANN) is helpful in attempting to differentiate normal mucosa from pathological lesions and leukoplakia from lichen planus (LP). The points in the plot (inside the cube) represent real clinical cases classified by the ANN based on texture analysis. Two-dimensional neural network predictions are shown on the walls of the cube (there are no sharp borders due to the application of fuzzy logic in the neural network mathematical engine): the purple surface area represents a diagnosis of leukoplakia, the orange surface area of LP and the green surface area of normal mucosa. Negative values of the co-occurrence factor combined with values higher than 0.3 of short run emphasis inverse moments (ShrtREmph) predicts a pathological lesion. When ShrtREmph is $0.3-0.8$ together with a value of less than -0.2 of the co-occurrence factor, the image should be considered to show leukoplakia. A positive value of the co-occurrence factor together with a low value of long run emphasis inverse moments (LngREmph) — approx. 0-40 - indicates normal oral mucosa. Lichen planus on that plane (left side wall) is presented when a higher value of LngREmph is noted. The extreme values of ShrtREmph (especially the lower extreme) combined with low values of LngREmph suggests normal mucosa (bottom of the cube). When the LngREmph value increases, a diagnosis of a pathological lesion should be strongly considered (generally LP pathology when LngREmph $>50$ )

The sensitivity for leukoplakia detection was $57 \%$, for LP detection it was $38 \%$ and for normal mucosa detection $94 \%$. The specificity of leukoplakia detection was $74 \%$, of LP $81 \%$ and of normal mucosa $88 \%$.

\section{Discussion}

Many parameters have been proposed to describe the microstructures found in medical scans. Fractal dimensions have been applied in the investigation of oral mucosa pathology, while run-length and co-occurrence matrices have been used in osteoporosis studies to describe bone before oral surgery. ${ }^{14,16-19}$ Due to the lack of publications on pathological oral mucosa lesion diagnosis with texture analysis, the 4 features previously used in bone healing research were introduced into this study. ${ }^{20,21}$

In leukoplakia, the normal, fine differences in brightness disappear and develop homogeneous white plates (Fig. 1). In LP, that process is similar, but the value of InvDfMom is lower (closer to normal mucosa) due to the pathological structure of the lesion, i.e., a mesh rather than a plate 
(Fig. 3). It seems that InvDfMom is an appropriate measure of pathological lesion creation in the oral mucosa which can be detected even in visible light. Obviously, automated differential diagnosis between LP and leukoplakia is still a challenge. Advanced mathematical techniques (PNN or FA) are only somewhat helpful.

Nowadays, digital light image analysis can be used to recognize a pathological lesion in the oral mucosa as presented above. When negative values of the co-occurrence factor are found along with higher values of ShrtREmph in imagery of the oral mucosa, then the physician should suspect a precancerous lesion in the area (Fig. 4).

This study indicates the need of search for alternatives to ensure proper access to healthcare and in partnership with non-specialized doctors from different macroregions of whole country. This research demonstrates the importance of using telemedicine, since it is a diagnostic method that allows for the early detection of oral pre-malignancy lesions, thus decreasing the number of unnecessary referrals to general dentists. ${ }^{22}$ Therefore, it helps to reduce not only wait times for face-to-face consultations, but also the costs associated with this process.

Many non-invasive systems such as ViziLite ${ }^{\circledR}$, ViziLite ${ }^{\circledR}$ PLUS, Velscope ${ }^{\circledR}$, and Identafi ${ }^{\circledR}$ are available to detect precancerous lesions. ${ }^{23}$ These systems are based on fluorescence or autofluorescence of suspicious lesions. Jain et al. confirmed that a method of ViziLite ${ }^{\circledR}$ PLUS examination was most effective in cases of leukoplakia in assessing the size, borders and shape of the lesion, followed by toluidine blue and incandescent light examinations. Methods using toluidine blue and ViziLite ${ }^{\circledR}$ PLUS examination demonstrated a sensitivity of $100 \%$ and a specificity of $97.3 \% .{ }^{24}$ Pallagatti et al. used toluidine blue to detect suspicious lesion staining in in vivo dysplastic cells. Toluidine blue is an acidophilic dye that selectively stains acidic tissue components such as DNA and RNA. Dysplastic lesions and in situ carcinomas contain much more DNA and RNA than the normal surrounding epithelium, so the use of in vivo toluidine blue staining may indicate premalignant or malignant lesions. ${ }^{25}$

Lalla et al. detected oral epithelial dysplasia using reflectance spectroscopy (Identafi, DentalEZ). Their results show that a system using violet light offered a sensitivity of $12.5 \%$ and a specificity of $85.4 \%$ for detecting oral epithelial dysplasia. ${ }^{26}$ Sambandham et al. applied a ViziLite ${ }^{\circledR}$ system to leukoplakia diagnosis. Their study shows that the sensitivity and specificity of ViziLite ${ }^{\circledR}$ are about $77.3 \%$ and $27.8 \%$, respectively. ${ }^{27}$ McIntosh et al. used a Microlux/DL system (AdDent Inc, Danbury, USA) for leukoplakia diagnosis and reported a sensitivity of $77.8 \%$ and a specificity of $70.7 \% .{ }^{28}$ Ibrahim et al. found that the sensitivity was $100 \%$ and the specificity was $32.4 \%$ in the visualization of suspicious premalignant lesions using Microlux/DL. ${ }^{29}$ In our study, the sensitivity of leukoplakia detection was $57 \%$, which was lower than in the abovementioned results, but the specificity of $74 \%$ was significantly higher than the ViziLite ${ }^{\circledR}$ application by Sambandham et al.

Honsi et al. used image cytometry to determine DNA ploidy in LP. The most common degree of DNA ploidy in LP lesions was diploidy. Comparing the 2 groups $\left(x^{2}\right.$ test of association, $\mathrm{p}=0.021$ ), they demonstrated that diploidy was associated with the reticular clinical form of LP, while aneuploidy was associated with the atrophic-erosive clinical form of oral LP. ${ }^{30}$

All of the abovementioned diagnostic methods are helpful in detecting LP and leukoplakia, but none of them is able to distinguish between these 2 types of lesions. Differential diagnosis of leukoplakia and LP in oral mucosa based on digital texture analysis in intraoral macrophotography is possible. Moreover, it can be used to develop computer/smartphone applications and can also be a helpful tool for general dentists to define the clinical problem before consultation with oral and maxillofacial surgeons.

\section{ORCID iDs}

Kamil Jurczyszyn (D) https://orcid.org/0000-0002-0667-7261

Marcin Kozakiewicz (D) https://orcid.org/0000-0001-9200-2828

\section{References}

1. Arora KS, Nayyar A, Kaur P, Arora KS, Goel A, Singh S. Evaluation of collagen in leukoplakia, oral submucous fibrosis and oral squamous cell carcinomas using polarizing microscopy and immunohistochemistry. Asian Pac J Cancer Prev. 2018;19(4):1075-1080.

2. Roopashree MR, Gondhalekar RV, Shashikanth MC, George J, Thippeswamy SH, Shukla A. Pathogenesis of oral lichen planus - a review. J Oral Pathol Med. 2010;39(10):729-734.

3. World Health Organization. World Health Organization classification of tumours. In: Barnes L, Eveson JW, Reichart P, Sidransky D, eds. Pathology and Genetics. Head and Neck Tumours. Lyon, France: International Agency for Research on Cancer Press; 2005:177-179.

4. Edwards P, Kelsch R. Oral lichen planus: Clinical presentation and management. J Can Dent Assoc. 2002;68(8):494-499.

5. Rennekampff HO, Fimmers R, Metelmann HR, Schumann H, Tenenhaus M. Reliability of photographic analysis of wound epithelialization assessed in human skin graft donor sites and epidermolysis bullosa wounds. Trials. 2015;28:235. doi:10.1186/s13063-015-0742-x

6. Chang WY, Huang A, Chen YC, et al. The feasibility of using manual segmentation in a multifeature computer-aided diagnosis system for classification of skin lesions: A retrospective comparative study. BMJ Open. 2015 May 3;5(4):e007823. doi:10.1136/bmjopen-2015-007823

7. Muehlematter UJ, Mannil M, Becker AS, et al. Vertebral body insufficiency fractures: Detection of vertebrae at risk on standard CT images using texture analysis and machine learning [published online as ahead of print on December 5, 2018]. Eur Radiol. 2018. doi:10.1007/ s00330-018-5846-8

8. Feng C, Lu F, Shen Y, et al. Tumor heterogeneity in gastrointestinal stromal tumors of the small bowel: Volumetric CT texture analysis as a potential biomarker for risk stratification. Cancer Imaging. 2018; 18:46. doi:10.1186/s40644-018-0182-4

9. Haralick R, Shanmugam K, Dinstein I. Textural features for image classification. IEEE Transactions on Systems Man and Cybernetics. 1973;3: 610-621.

10. Dash M, Liu H. Feature selection for Classification (1997) Elsevier Science Inc., https://www.sciencedirect.com/science/article/pii/ S1088467X97000085

11. Szczypiński P, Strzelecki M, Materka A. MaZda - a software for texture analysis. Proceedings of ISITC 2007, November 23-23, 2007, Jeonju, Republic of Korea: 2007:245-249.

12. Szczypiński P, Strzelecki M, Materka A, Klepaczko A. MaZda - a software package for image texture analysis. Comput Methods Programs Biomed. 2009;94(1):66-76. 
13. Hadrowicz J, Hadrowicz P, Gesing A, Kozakiewicz M. Age dependent alteration in bone surrounding dental implants. Dent Med Probl. 2014; 51(1):27-34.

14. Kołaciński M, Kozakiewicz M, Materka A. Textural entropy as a potential feature for quantitative assessment of jaw bone healing process. Arch Med Sci. 2015;11(1):78-84

15. Kozakiewicz M, Hadrowicz P, Hadrowicz JM, Gesing A. Can torque force during dental implant placement combined with bone mineral density of lumbar spine be prediction factors for crestal bone structure alterations? Dent Med Probl. 2014;51(4):448-457.

16. Jurczyszyn K, Kubasiewicz-Ross P, Nawrot-Hadzik I, Gedrange T, Dominiak M, Hadzik J. Fractal dimension analysis a supplementary mathematical method for bone defect regeneration measurement. Ann Anat. 2018;219:83-88.

17. Jurczyszyn K, Kazubowska K, Kubasiewicz-Ross P, Ziółkowski P, Dominiak M. Application of fractal dimension analysis and photodynamic diagnosis in the case of differentiation between lichen planus and leukoplakia: A preliminary study [published online as ahead of print on August 7, 2018]. Adv Clin Exp Med. 2018. doi:10.17219/ acem/80831

18. Benhamou CL, Poupon S, Lespessailles E, et al. Fractal analysis of radiographic trabecular bone texture and bone mineral density: Two complementary parameters related to osteoporotic fractures. J Bone Miner Res. 2001;16(4):697-704.

19. Lespessailles $E$, Gadois C, Kousignian I, et al. Clinical interest of bone texture analysis in osteoporosis: A case control multicenter study. Osteoporos Int. 2008;19(7):1019-1028.

20. Kozakiewicz M, Stefańczyk M, Materka A. Krótkie łańcuchy podobnych punktów obrazu w radioteksturze kości wyrostka zębodołowego szczęki i części zębodołowej żuchwy u człowieka. PolMerk Lek. 2007;133:200-205.
21. Kozakiewicz M, Stefańczyk M. Ocena regeneracji ubytków kości zaopatrzonych materiałem HA-Biocer z zastosowaniem obiektywnej miary matematycznej - macierzy długości serii. Mag Stomat. 2010;23(6):77-82

22. Piccoli MF, Amorim BD, Wagner HM, Nunes DH. Teledermatology protocol for screening of skin cancer. An Bras Dermatol. 2015;90(2): 202-210. doi:10.1590/abd1806-4841.20153163

23. Mascitti M, Orsini G, Tosco V, et al. An overview on current non-invasive diagnostic devices in oral oncology. Front Physiol. 2018;9:1510. doi:10.3389/fphys.2018.01510

24. Jain N, Nagarajappa AK, Bhasin M, Pandya D, Tripathi KP. Role of chemiluminescence examination as non-invasive diagnostic tool in early detection of leukoplakia. J Oral Biol Craniofac Res. 2018;8(3):177-181. doi:10.1016/j.jobcr.2017.04.004

25. Pallagatti S, Sheikh S, Aggarwal A, et al. Toluidine blue staining as an adjunctive tool for early diagnosis of dysplastic changes in the oral mucosa. J Clin Exp Dent. 2013;5(4):187-191. doi:10.4317/jced.51121

26. Lalla Y, Matias MA, Farah CS. Assessment of oral mucosal lesions with autofluorescence imaging and reflectance spectroscopy. J Am Dent Assoc. 2016;147(8):650-660. doi:10.1016/j.adaj.2016.03.013

27. Sambandham T, Masthan KM, Kumar MS, Jha A. The application of vizilite in oral cancer. J Clin Diagn Res. 2013;7(1):185-186. doi:10. 7860/JCDR/2012/5163.2704

28. McIntosh L, McCullough MJ, Farah CS. The assessment of diffused light illumination and acetic acid rinse (Microlux/DL) in the visualisation of oral mucosal lesions. Oral Oncol. 2009;45(12):e227-e231. doi:10.1016/j.oraloncology. 2009.08.001

29. Ibrahim SS, Al-Attas SA, Darwish ZE, Amer HA, Hassan MH. Effectiveness of the Microlux/DLTM chemiluminescence device in screening of potentially malignant and malignant oral lesions. Asian Pac J CancerPrev. 2014;15(15):6081-6086.

30. Hosni ES, Yurgel LS, Silva VD. DNA ploidy in oral lichen planus, determined by image cytometry. J Oral Pathol Med. 2010;39(3):206-211. doi:10.1111/j.1600-0714.2009.00833.x 